\title{
La historia clínica y el examen físico son útiles para el diagnóstico de enfermedad pulmonar obstructiva crónica
}

The accuracy of patient history, wheezing, and laryngeal measurements in diagnosing Obstructive Airway Disease. Straus S, Mc Alister F, Sackett D et al. JAMA. 2000 april 12; 283:1853-7.

\section{Objetivo}

Determinar la precisión de algunos aspectos de la historia clínica y el examen físico para el diagnóstico de enfermedad pulmonar obstructiva crónica (EPOC).

Diseño

Estudio de corte transversal.

\section{Lugar}

Estudio muticéntrico realizado en 14 países. Incluyó centros de atención primaria $(50 \%)$ y centros de segundo nivel $(50 \%)$.

\section{Pacientes}

Se incluyeron 332 pacientes en tres categorías: pacientes que se sabían EPOC (25\%), pacientes que se sospechaba EPOC (37\%) y pacientes que no se sabían ni se sospechaba EPOC (39\%). Los 309 pacientes que se analizaron (se excluyeron 23 por asma previo) tenían una edad media de 56 años y el $43 \%$ eran mujeres. El $39 \%$ de los pacientes analizados fueron no fumadores.

Descripción de los test y el test de referencia (gold standard) autoreporte del paciente como EPOC, el grado de tabaquismo (paquetes/año), la presencia de sibilancias y la altura laríngea medida desde el cartílago tiroides al hueco supraesternal. La altura laríngea máxima se midió al final de la espiración, y la mínima al final de la inspiración. Se definió como descenso laríngeo a la diferencia entre la máxima y mínima longitud laríngea. A todos los pacientes incluidos se les realizó una espirometría. El operador que realizaba la espirometría desconocía los resultados del examen clínico.

\section{Medición de los resultados}

Se calcularon la sensibilidad, especificidad y los coeficientes de probabilidad ("likelihood ratios")* de las variables estudiadas. Las variables de la historia clínica estudiadas fueron: la edad, el

\section{Resultados Principales}

De los 309 pacientes analizados 162 (52\%) tuvieron una espirometría con criterios de EPOC. La altura laríngea mínima y máxima fue menor en pacientes que cumplían criterios espirómétricos de EPOC $(3,8 \mathrm{~cm}$ vs $4.6 \mathrm{~cm}$, y $5,4 \mathrm{~cm}$ vs $6,3 \mathrm{~cm}$, ambas $p<0,01$ ). El descenso laríngeo no se asoció al diagnóstico de EPOC. Los hallazgos que predicen EPOC del análisis multivariado* se reportan en la Tablas 1 y 2 .

Tabla 1. Coeficientes de probabilidad ajustados de todos los pacientes analizados $\mathrm{N}=309$

\begin{tabular}{l|c|c}
\hline Variable estudiada & $\begin{array}{c}\text { Variable Presente } \\
\text { Coeficiente de } \\
\text { probabilidad positivo }\end{array}$ & $\begin{array}{c}\text { Variable Ausente } \\
\text { Coefieinte de } \\
\text { probabilidad negativo }\end{array}$ \\
\hline 1. Auto-reporte de EPOC & 7.3 & 0.5 \\
\hline 2. Tabaquismo > de 40 paquetes/año & 8.3 & 0.8 \\
\hline 3. Edad $\geq$ de 45 años & 1.3 & 0.4 \\
\hline 4. Altura laríngea máxima = de $4 \mathrm{~cm}$ & 2.8 & 0.8 \\
\hline Las 4 variables combinadas & 220.5 & 0.13 \\
\hline
\end{tabular}

Tabla 2. Coeficientes de probabilidad ajustados sin incluir a los pacientes EPOC conocidos $\mathrm{N}=233$

\begin{tabular}{l|c|c} 
Variable estudiada & $\begin{array}{c}\text { Variable Presente } \\
\text { Coeficiente de } \\
\text { probahilidad positivo }\end{array}$ & $\begin{array}{c}\text { Variable Ausente } \\
\text { Coeficinte de } \\
\text { probahilidad negativo }\end{array}$ \\
\hline 1. Tabaquismo $>$ de 40 paquetes/año & 11.6 & 0.9 \\
\hline 2. Edad $\geq$ de 45 años & 1.4 & 0.5 \\
\hline 3. Altura laríngea máxima $=$ de $4 \mathrm{~cm}$ & 3.6 & 0.7 \\
\hline Las 3 variables combinadas & 58.5 & 0.32 \\
\hline
\end{tabular}

\section{Conclusiones}

La edad mayor de 45 años, fumar más de 40 paquetes/año, la altura laríngea $=$ a $4 \mathrm{~cm}$ al final de la espiración y el autoreporte de EPOC, son los datos de la historia clínica y el examen físico que mayor rendimiento diagnóstico tienen para EPOC. Las sibilancias y el descenso traqueal son de escaso valor diagnóstico.

Fuente de financiamiento: No referida.

\section{Comentario}

Si bien es cierto hacen falta estudios que validen el modelo de predicción (como concluyen los autores), este artículo aporta una herramienta diagnóstica de utilidad para la práctica cotidiana. En línea general, el examen clínico aporta escasos signos y síntomas lo suficientemente sensibles y precoces como para hacer diagnóstico temprano de EPOC. Lamentablemente, la gran mayoría de los diagnósticos de EPOC, se hacen cuando el paciente consulta con síntomas (disnea de esfuerzo o tos crónica) y a esta altura el grado de deterioro es de moderado a avanzado.

Por tal motivo si queremos hacer prevención primaria de la EPOC, es fundamental trabajar sobre el tabaquismo antes que empiecen los síntomas y así evitar el principal factor de riesgo para desarrollarla.

Los resultados de este estudio se reportan con un valor denominado "Coeficiente de Probabilidad" el cual expresa cuántas veces más probable es encontrar ese resultado en un EPOC que encontrarlo en un no-EPOC. Tomando un ejemplo del presente estudio que se observa en la tabla 2: un sujeto mayor de 45 años que fuma más de 40 paquetes año y que tiene una altura laríngea $=$ a $4 \mathrm{~cm}$ (es decir que tiene las 3 variables estudiadas presentes), tiene 58.5 veces más chance de ser un paciente EPOC que de no serlo. El coeficiente de probabilidad tiene gran utilidad clínica, ya que brinda información valiosa acerca de cómo interpretar el resultado de un test diagnostico y es independiente de la probabilidad previa de la patología. En pacientes que reúnan estas características, con alto rendimiento diagnóstico, prácticamente se podría prescindir de la espirometría para el diagnóstico de EPOC

Sin embargo en la EPOC, la utilidad de la espirometría no es sólo diagnóstica. Una de las utilidades que cumple, es en el monitoreo de la respuesta a los nuevos tratamientos. Es sabido que ciertas drogas que se utilizan en el manejo de la EPOC (ej. aminofilina o corticoides) tienen efectos adversos serios y por lo tanto se aconseja documentar objetivamente la respuesta espirométrica cuan-

Otra utilidad de la espirometría en el manejo de la EPOC es con fines pronósticos. Numerosos estudios mostraron que existe relación entre los valores espirométricos de los pacientes con EPOC y la tasa de sobrevida (tanto durante como fuera de la exacerbación). 1-3

Como conclusión final es importante destacar que este estudio corrobora algo que ya se sabia previamente: 4 el factor predictor más poderoso en el examen clínico del paciente, excluyendo el autoreporte de EPOC, es el grado de tabaquismo. do se las utiliza.

Dr. Pablo Jinich [ Unidad de Medicina Familiar y Preventiva. Hospital Italiano de Buenos Aires ]

*Ver glosario

Referencias

1. Thomason MP, Strachan DP y col. Which spirometric indices best predict subsequent death from chronic ostructive pulmonary disease? Thorax 2000;55(9):785-8.

2. Traver GA, Cline MG y col. Predictors of mortality in chronic obstructive pulmonary disease. A 15 year follow up study. Am Rev Respir Dis 1979:119(6):895-902.

3. Niewoehner DE, Collins D y col. Relation of FEV1 to clinical outcomes during exacerbations of chronic obstructive pulmonary disease. Am Rev Respir Dis 2000;161(4):1201-5.

4. Holleman DR Jr. Simel DL. Does the clinical examination predict airflow limitation?. JAMA 1995; 273(4):313-9. 